Service social

\title{
Travail social de groupe et intervention de réseaux. Spécificités et complémentarités
}

\section{Christiane Besson}

Volume 46, numéro 2-3, 1997

Groupes - Symposium 1997

URI : https://id.erudit.org/iderudit/706763ar

DOI : https://doi.org/10.7202/706763ar

Aller au sommaire du numéro

Éditeur(s)

École de service social de l'Université Laval

ISSN

1708-1734 (numérique)

Découvrir la revue

Citer cet article

Besson, C. (1997). Travail social de groupe et intervention de réseaux.

Spécificités et complémentarités. Service social, 46(2-3), 127-145.

https://doi.org/10.7202/706763ar d'utilisation que vous pouvez consulter en ligne.

https://apropos.erudit.org/fr/usagers/politique-dutilisation/ 


\section{Travail social de groupe et intervention de réseaux Spécificités et complémentarités}

Christiane BESSON

Venant d'un autre pays et participant d'une autre culture, j'estime utile de décrire d'abord brièvement le contexte où je vis. Cela n'est pas sans influencer ma conception de l'intervention de réseaux, aussi bien que celle que j'ai du travail social de groupe. Je présenterai ensuite quelques observations concernant la confusion qui entoure certains concepts, m'efforçant de mieux les définir avec leurs spécificités, leurs similitudes et leurs différences ainsi que leurs complémentarités. Je terminerai en indiquant quelques possibilités de passage d'une méthode à l'autre, possibilités qui conduisent à des interventions mixtes.

\section{LE CONTEXTE OÙ JE VIS : UN MILIEU PLURICULTUREL...}

La Suisse se trouve au carrefour de trois cultures et de quatre langues nationales. Lorsque vous arrivez en gare de Berne, capitale de notre pays, vous trouvez les indications essentielles écrites en langues allemande, française, italienne et romanche (langue ancienne issue du latin); vous entendez les trains annoncés en deux langues, l'allemand et le français, souvent aussi (en plus) en anglais. Vingt cantons et six demi-cantons s'étendent du Jura aux Alpes en passant par le plateau. Chacun d'eux a son histoire et ses traditions, son folklore, son architecture et son économie, souvent même ses dialectes. La Suisse abrite bientôt 7 millions d'habitants, répartis 
très inégalement sur 41107 kilomètres carrés; deux tiers de cette surface étant formés de montagnes inhabitables, la densité est énorme, soit 158 habitants au kilomètre carré. Le chômage est aussi inégalement réparti.

\section{... ET POLITIQUEMENT ORIGINAL}

Une des particularités de la Suisse est sa constitution progressive en Confédération d'États, pour la plupart à l'origine indépendants. L'histoire remonte au XIII siècle (août 1291), au moment où trois petites communautés voisines concluaient, par l'intermédiaire de trois hommes dont les noms sont restés dans les mémoires - Walter Fürst, Arnold de Melchtal et Werner Stauffacher -, une alliance militaire et politique, économique aussi à certains égards, qui constitue le noyau de notre pays. Aux trois cantons primitifs se sont ajoutés, peu à peu, au cours des ans, de nouveaux États, jusqu'au milieu du siècle dernier, moment où la Confédération a cessé de s'étendre sur le plan géographique. Tout au long de cette période, l'alliance s'est étendue et renforcée, les États membres abandonnant progressivement à la Confédération un nombre croissant de domaines d'activité. II n'en demeure pas moins que les 26 cantons et demicantons qu'elle comprend aujourd'hui conservent des compétences étendues, parfois exclusives, notamment dans le domaine de l'instruction publique. Dans tous les domaines qui n'ont pas été délégués à la Confédération, leur souveraineté prime celle de l'État central. Il faut dire aussi que la cellule de base de l'organisation politique, dans presque tous les cantons, est la commune, qui a gardé (c'est une particularité probablement unique en Europe) un certain nombre de prérogatives de l'époque médiévale, où celles-ci furent à l'apogée de leur vitalité, notamment le droit de prélever des impôts (24 \% environ de la masse fiscale). Avant même d'être citoyen suisse, le citoyen est toujours originaire d'une commune, celle de sa famille. Je mentionne enfin une dernière particularité (il y en aurait bien d'autres...), qui est celle d'appeler fréquemment les citoyens aux urnes, non seulement pour élire leurs magistrats, mais pour prendre toutes sortes de décisions (c'est ce qu'on appelle la « démocratie directe $")$.

De cette histoire et de cette culture découle le fait que l'idée d'unité dans la diversité constitue un principe fondamental dans notre 
pays, où chaque région a su conserver ses particularités dans les domaines linguistique, culturel et confessionnel, sociologique, économique et politique.

Cela crée évidemment des disparités, des inégalités et des originalités d'un canton à l'autre, sur le plan des institutions sociales par exemple.

\section{CaRActères GÉnÉrauX du traVAIL SOCIAL EN SUISSE}

Ne croyez pourtant pas que le travail social échappe, en Suisse romande, à l'influence des courants de réflexion qui, soufflant de partout, conduisent à l'engager sur des voies nouvelles. Mais ces courants restent très étroitement liés à un pragmatisme foncier qui est l'un des traits essentiels de l'Helvète au travail... Je résumerai ici quelques-unes des particularités principales du travail social dans notre pays, en indiquant notamment les suivantes :

- la diversité et la spécialisation des institutions correspondant aux diversités régionales, et dont l'origine est dans beaucoup de cas due à l'initiative privée, surtout en Suisse romande;

- la taille des institutions, en général petite à moyenne : dans mon canton, le Pays de Vaud, quatre à cinq institutions seulement accueillent plus de cent personnes handicapées, les autres dépassant rarement la trentaine; les maisons d'enfants ou de jeunes en difficulté sont en général moins grandes encore. Un service social occupant une centaine de collaborateurs est considéré comme un immense service... il en existe peut-être quatre en Suisse romande, les autres occupant moins de cinquante professionnels;

- la priorité qu'on donnera presque toujours à l'action plus qu'à l'écrit;

- l'autonomie que revendiquent les intervenants sociaux et les équipes dans leur travail;

- $\quad$... et par conséquent la méfiance viscérale (démocratie directe oblige...) par rapport à la hiérarchie;

- les tendances actuelles qui s'orientent principalement vers le maintien à domicile, la prévention des maltraitances, le travail 
centré sur les effets d'un chômage en augmentation, le travail de rue, les familles à risque et les SDF (sans domicile fixe), activités souvent réalisées pour commencer sous forme de " projets pilotes" menés dans des "zones pilotes".

\section{La formation et le perfectionnement des travailleurs sociaux en Suisse}

Je terminerai la présente section en évoquant la situation dans ce domaine de la formation professionnelle en Suisse romande, c'està-dire d'expression française; on y compte six cantons, qui réunissent un cinquième de la population du pays (soit un million de personnes environ). Quelques mots avant tout au sujet des formations de base.

La formation en travail social y est d'abord délivrée, tantôt en cours d'emploi, tantôt à plein temps, au sein d'écoles appelées généralement aujourd'hui "Écoles supérieures d'études sociales". Les plus anciennes ont été créées au début de notre siècle, les dernières se sont développées au cours des trente dernières années, pratiquement toutes à partir d'une initiative privée. Aujourd'hui, les communes (parfois), les cantons, souvent, et presque toujours la Confédération les subventionnent. La malice des temps conduit à certaines remises en question, en particulier du côté fédéral.

Une seule des quatre universités de Suisse romande, celle de Fribourg, offre à la Chaire de travail social des études à la fois théoriques et pratiques qui aboutissent à une licence en travail social et permettent d'accéder au doctorat. À Fribourg aussi, on trouve un Institut de pédagogie curative formant des éducateurs et des enseignants spécialisés qui peuvent accéder à un diplôme ou à une licence. Nous ne connaissons pas de formations moins spécialisées, telles celle des techniciens en travail social qui, chez vous, sortent du cégep ou celle des moniteurs éducateurs à la française.

Quant à la formation continue, elle s'est développée depuis une vingtaine d'années, d'abord à l'initiative de l'Institut d'Études sociales de Genève qui a ouvert la voie par la création du Céfoc, Centre d'études et de formation continue. D'autres centres se sont créés par la suite en Suisse romande, rattachés soit tantôt aux écoles, tantôt aux associations professionnelles, soit sous l'impulsion d'un professionnel à titre privé. 
Un tout nouveau diplôme supérieur en travail social peut être obtenu à l'Université de Neuchâtel. Ce cours, mis sur pied grâce à la collaboration des différentes écoles de service social et de certaines associations professionnelles, est ouvert à des professionnels diplômés et chevronnés. L'Université de Genève offre la possibilité d'obtenir un certificat en " politique sociale » et en " histoires de vie ".

On le voit, sur bien des points, les conditions de la formation professionnelle en Suisse romande diffèrent de celles qu'on trouve, à ce que je crois savoir, au Québec. La recherche officielle, par exemple, était réservée jusqu'il y a très peu de temps aux chercheurs universitaires avec lesquels les travailleurs sociaux n'entretenaient guère de relations. C'est un phénomène relativement récent de voir les Écoles supérieures de travail social se joindre aux recherches du Fonds national de la recherche scientifique. L'Institut d'Études sociales de Genève a mis en place depuis une vingtaine d'années un secteur de recherche qui s'intéresse particulièrement à des questions propres au travail social et publie les meilleurs travaux de recherche menés par les étudiants. II est rare de voir se constituer, comme chez vous, une équipe de praticiens et de chercheurs travaillant ensemble autour d'un projet subventionné. Ce qu'on commence à voir, non sans quelque inquiétude, c'est la mise au travail par l'État (en général sur le plan cantonal) d'équipes de chercheurs engagés dans des enquêtes à caractère scientifique..., mais dont on soupçonne qu'elles visent souvent aussi à déboucher sur des économies. C'est, bien entendu, la situation conjoncturelle qui en est la cause...

Alors ils s'aimeront de s'épauler l'un l'autre et de bâtir ensemble. Antoine de Saint-Exupéry

\section{QUELQUES PARALLÈLES DANS LE DÉVELOPPEMENT DU TRAVAIL SOCIAL DE GROUPE ET DE L'INTERVENTION DE RÉSEAUX}

Les réflexions qui suivent ne visent pas à établir une hiérarchie entre le travail social de groupe (tsg) et l'intervention de réseaux, ni à dresser une simple liste des différences et des ressemblances. Nous tentons d'y esquisser, par comparaisons successives, la nature des relations qui peuvent exister entre ces deux pratiques. 
La comparaison n'est donc pas une fin en soi, mais elle nous permettra de mettre en évidence les spécificités de chacune des deux approches, d'établir des passerelles, d'imaginer des articulations, de les combiner finalement l'une à l'autre pour offrir aux clients un service approprié, adaptable et de qualité. Nombreux sont parmi les travailleurs sociaux ceux qui, préoccupés par l'importance des problèmes actuels, désirent élargir leur écoute, démystifier leur rôle et concentrer leur énergie sur l'autonomie des groupes avec lesquels ils travaillent.

Je limiterai ici ma réflexion au seul domaine des pratiques collectives, au sens large, qui comprend les « jumelages », le travail avec des groupes en service social, l'organisation communautaire et l'intervention de réseaux. Ces pratiques appartiennent toutes à la famille des formes d'actions sociales promotionnelles, favorisant le pôle " population » au détriment du pôle « institution », laissant une place privilégiée aux clients pour définir leurs problèmes et tenter de les résoudre.

Commençons par quelques définitions, en nous rappelant que

Ce qui est simple est faux, ce qui ne l'est pas est inutilisable.

Paul Valéry

\section{Définitions et précisions terminologiques}

\section{Le travail social de groupe}

\section{Le groupe restreint}

On appelle groupe restreint un ensemble de personnes réunies, pour un temps donné, dans un même lieu en vue de partager un objectif commun, dans une relation en face à face et en interagissant les unes avec les autres.

\section{Étymologiquement}

Le mot se rattache à une racine germanique, kruppa, masse arrondie, évoquant l'idée de cercle; j'y vois l'évocation du côté symbolique que revêt la rencontre en groupe, avec les processus inconscients qu'elle peut susciter. 
Les Italiens ont utilisé la même racine pour forger un terme technique, dans le domaine des beaux-arts; gruppo y désigne un ensemble de personnages réunis dans un tableau ou une sculpture. En observant un tableau ou un bas-relief, les attitudes sont révélatrices des relations que l'artiste a voulu illustrer; la pose des acteurs ou des figurants, leurs attitudes, le mouvement de leur corps ou de leurs vêtements, l'expression de leur visage, la direction même de leur regard, tout cela rend perceptibles leurs intérêts, la nature des liens qui les rassemblent, l'organisation, le contexte, voire le but poursuivi par le groupe.

On peut même établir un lien entre ces deux images et les deux courants fondamentaux de pensée centrés sur les groupes restreints que sont, d'une part, celui de la psychanalyse, qui met en lumière l'affectivité et l'inconscient du groupe avec Freud, Klein, Bion, Moreno, Anzieu, Pages, etc., et, d'autre part, le courant psychosociologique centré sur la communication et l'efficacité avec Lewin, Porter, Bavelas, Lippit et White, etc.

Du point de vue de la pratique, le travailleur social verra dans le même sens défiler dans sa mémoire deux catégories de groupes de clients, les premiers centrés sur eux-mêmes, sur le développement personnel, l'expression des sentiments et l'entraide, les seconds ouverts sur l'extérieur, engagés dans l'accomplissement de tâches, de réalisations, de défense des droits.

\section{Débuts et développement du travail social de groupe (tsg)}

Le tsg se rattache à une longue tradition de pratiques et d'enseignements, puisque les premiers cours de travail social de groupe ont été donnés en 1928 à Cleveland, les premiers pas de l'association pour le développement du travail social aux Etats-Unis datant de 1936. En Europe l'impulsion a été donnée par l'Organisation des Nations Unies dans les années 1940, tant sur le plan du tsg, d'ailleurs, que sur celui des autres méthodes de travail social. Dès cette époque aussi l'enseignement du tsg fit sa place dans lesécoles suisses, d'abord à Zurich, puis dès les années 1970 en Suisse romande.

\section{But du travail social de groupe}

Aider les gens à faire partie d'un groupe qui signifie quelque chose pour eux, de telle sorte qu'ils finissent eux-mêmes par signifier quelque chose pour le groupe... 
Aider les gens dans leurs relations avec les autres et avec leur milieu ne signifie pas les ajuster dans le sens d'une acceptation passive du statu quo par l'individu qui doit apprendre à vivre dans une situation donnée. Cela signifie plutôt que les gens, individus ou groupes, doivent savoir quand ils doivent changer eux-mêmes et quand il leur faut modifier leur entourage humain, économique ou politique.

Gisella Konopka

\section{Définitions et précisions terminologiques L'intervention de réseaux}

\section{Le réseau primaire}

Le réseau primaire est un ensemble de personnes qui se connaissent et sont unies par des liens de parenté, d'amitié, de voisinage et de travail.

\section{Le réseau secondaire}

Le réseau secondaire est constitué par les institutions qui produisent des services.

\section{Que nous racontent les mots?}

Filet, tissu à mailles très larges, tel est à l'origine le sens du mot réseau en vieux français. On pense alors au filet du pêcheur, source d'abondance, à celui du trapéziste, qui amortit la chute. Le tissu devient tissage, et ne dit-on pas tisser des liens? et si l'on songe à la dentelle, on évoque la toile d'araignée, encore une forme de piège. Deux catégories donc de réseaux, ceux qui lient pour le meilleur ou pour le pire, ceux qui libèrent et ceux qui enferment, favorisent les communications ou les paralysent, font circuler l'énergie ou la neutralisent.

La diversité des usages que l'on fait du mot réseau illustre la complexité de l'organisation et des rapports sociaux, caractérisés aussi par l'ouverture, la mouvance, la variabilité des contacts humains et un certain flou. C'est pourquoi les réseaux sociaux sont parfois difficilement repérables, ce qui complique souvent la tâche tant des chercheurs que des praticiens. 


\section{Débuts et développement de lintervention de réseaux}

Ce sont des anthropologues et chercheurs britanniques (Bott et Barnes) qui ont les premiers exploité et développé la notion de réseaux dans les années 1950. Leurs travaux ont été repris et prolongés par des sociologues américains, italiens et français. Sous l'impulsion de Ross Speck, psychiatre nord-américain, travaillant à partir des écrits de Barnes dont il avait eu connaissance grâce à Irving Goffmann, se constituèrent et se développèrent les thérapies de réseaux dans le contexte médical. Par la suite, dès les années 1970 au Québec, est apparue l'intervention de réseaux proprement dite, essentiellement avec les travaux de l'équipe de Claude Brodeur et Richard Rousseau. En Europe, particulièrement en France, en Italie, en Belgique et en Suisse, ces pratiques se sont développées dès les années 1980 dans le champ du travail social et éducatif.

Le projet d'une intervention de réseaux

Le projet d'une intervention de réseaux, dès qu'on en fait la synthèse, consiste ainsi à redonner au réseau primaire à la fois sa dimension collective et son pouvoir de connaître et de régler ses propres problèmes...

Au terme du processus, c'est tout un collectif qui se penche sur l'ensemble de ses problèmes aussi bien que sur des problèmes particuliers, afin d'en assumer l'entière responsabilité, quitte à demander à l'institution un support temporaire et des services techniques.

Claude Brodeur

\section{LE TRAVAIL SOCIAL DE GROUPE}

DANS SES RAPPORTS AVEC L'INTERVENTION DE RÉSEAUX

\section{Deux modalités différentes d'existence du collectif ... constitué artificiellement}

Le groupe en tsg est formé par le professionnel, qui en fixe la composition et décide s'il sera appelé à s'ouvrir ou restera fermé. II est réuni par lui, selon un rythme convenu, avec des règles précises; les rencontres ont lieu en principe dans un des locaux de son institution. Lorsque le travail commence, les membres du groupe ne 
partagent pas d'histoire commune; ils vont construire cette histoire ensemble. Progressivement, l'entraide et la prise d'initiative étant encouragées, les relations se développent en d'autres temps et en d'autres lieux que ceux des réunions formelles. Les événements qui surviennent dans la vie personnelle de chacun n'influencent le travail du groupe que s'ils sont partagés, permettant une réflexion sur le chemin accompli, ici et aujourd'hui, pour en tirer les conséquences, ailleurs et pour demain, ensemble ou individuellement.

\section{... reconnu dans son existence naturelle}

Le réseau est au contraire un groupe naturel. Il existe et vit dans un lieu donné, même si ce lieu correspond à un territoire psychique et affectif plus que géographique. Avant même l'intervention d'un travailleur social, les membres du réseau ont établi entre eux des liens, souvent plus affectifs que fonctionnels. Ils partagent une histoire et un vécu communs, qui se poursuivront au-delà du travail entrepris avec l'intervenant. C'est lui qui se déplace dans les endroits où les membres du réseau veulent bien le recevoir; et ce sont eux qui décident qui sera invité lors de chaque séance. L'intervenant peut également, parfois, proposer d'accompagner tel ou tel membre du réseau dans un autre endroit, par exemple l'hôpital, la prison, une autre ville. Le réseau n'est pas nécessairement présent chaque fois dans son ensemble, suivant les circonstances et les besoins. Ce n'est pas sans signification de se déplacer, de se trouver sur le terrain des clients, dans leur maison, leur cuisine; il faut créer la confiance nécessaire. Le réseau continue à vivre en dehors des rencontres avec l'intervenant; c'est donc tout naturellement que les événements quotidiens ont des répercussions directes sur la situation et les relations dans le réseau, ce qui accentue encore le caractère mouvant et insaisissable de celui-ci. Tout cela relativise l'action du travailleur social pour donner plus de poids à ce qui se trame dans le réseau.

\section{QUELQUES ASPECTS COMMUNS DANS LA PRATIQUE DU TSG ET DE L'INTERVENTION DE RÉSEAUX}

\section{Tous deux ont affaire au collectif...}

Un premier point commun tient au fait que les deux formes d'intervention s'adressent au " collectif », à plusieurs personnes en relation, 
sans négliger pour autant la personne en tant que membre ayant une place particulière au sein de ce collectif. C'est ce collectif comme tel qui est considéré comme le " client » du travailleur social, composé par un ensemble de personnes que concernent les mêmes actions, les mêmes questions, les mêmes problèmes, partageant un sentiment d'appartenance, exprimé par un "nous ", parfois mobilisé à travers une action.

\section{... tantôt conscient, tantôt inconscient}

La dimension de l'inconscient collectif peut se révéler tant sur le plan du groupe que sur celui des réseaux. Les travailleurs qui parviennent à s'y intéresser et à s'y repérer sans perdre de vue ni leur mandat ni leur position montreront d'autant plus de compréhension et d'efficacité.

\section{L'impulsion est donnée par le travailleur social}

Il est rare que la demande soit formulée par le groupe ou le réseau comme tel; le travailleur social est généralement mobilisé par une demande individuelle, qui l'incite à partir à la rencontre du groupe ou des réseaux, là où ils se trouvent, essentiellement chez les personnes elles-mêmes.

\section{Un élargissement du regard est nécessaire...}

Les deux intervenants doivent faire preuve d'une écoute et d'un regard particuliers. En effet, chaque groupe, chaque réseau a son visage particulier et nécessite une nouvelle approche. De plus, il faut tantôt anticiper la possibilité d'une mise en commun des problèmes analogues au sein du groupe et motiver les membres potentiels pour qu'ils y participent; tantôt discerner le réseau dans le discours individuel du client, puis s'en faire accepter comme intervenant.

\section{... ainsi qu'une grande souplesse dans l'organisation du travail}

Le travailleur social de groupe, comme l'intervenant de réseaux, fait preuve de souplesse dans l'aménagement de son horaire, les rencontres ayant souvent lieu en dehors des heures de bureau, afin 
que les membres du groupe et des réseaux puissent être présents. Les deux sont appelés à s'engager de façon personnelle à l'égard du collectif qui compte sur eux dans la durée et la régularité. Tous deux partagent par exemple des moments de fête, des réunions plus larges si cela est souhaité et si cela concourt à la poursuite des objectifs.

\section{L'intervention vise l'« autonomie du collectif "...}

L'autonomie du groupe ou du réseau en tant que collectif est la caractéristique essentielle du but visé par les intervenants. II s'agit de mettre en œuvre quelques valeurs essentielles telles que l'autonomie, l'autodétermination, la participation, la solidarité, le respect, la répartition des forces et des pouvoirs, atteignant au cœur de l'humain les questions existentielles de l'identité, de l'appartenance et des liens.

Comme l'ont vérifié tous ceux qui s'y sont engagés, un groupe ou un réseau n'est jamais d'emblée ni autonome ni responsable; il ne s'engage pas sans un peu de méfiance dans un travail commun; il y faut de l'énergie, de la persévérance et de la régularité, même si l'on travaille à court terme, même si l'on intervient de manière ponctuelle.

L'intervenant acquiert alors une capacité à anticiper celle des participants ou du collectif comme tel à progresser vers cette autonomie. II favorise les communications entre les membres du groupe ou du réseau, l'exercice de la prise de parole et la collaboration. II invite à la polyvalence des rôles et des activités: les membres du groupe apprennent à utiliser à leur tour les techniques qu'ils ont acquises à travers leur expérience et leur formation. Travailler en commun ne les effraie plus, mais suscite au contraire leur énergie. Ils partagent le goût du risque, l'attirance pour la nouveauté.

\section{... les limites de l'« autonomisation à tout prix »}

Dans les deux formes d'intervention, l'attitude du professionnel bien intentionné peut conduire au paradoxe de vouloir autonomiser et collectiviser à tout prix. Ce danger ne peut être évité que si l'intervenant se montre capable d'une attention suffisante pour percevoir le message, explicite ou implicite, que le groupe ou le réseau lui adresse et qui précise ce vers quoi ils tendent et à quel stade ils veulent 
s'arrêter. N'y aurait-il pas un certain angélisme à oublier, en travaillant avec des collectifs, que les rapports de force, de pouvoir et de savoir y coexistent de toute façon et que le travailleur social, de par sa position, y est intimement mêlé.

\section{... Quelques similitudes méthodologiques}

En travail social de groupe comme en intervention de réseaux les intervenants doivent connaître les phases du développement d'un groupe ou celles de l'intervention de réseaux, dont le tableau a été établi à partir de nombreuses expériences d'interventions; ainsi pourront-ils se repérer dans l'évolution du groupe et du réseau, exercer aussi un certain contrôle sur leur travail.

\section{Le client devient un partenaire actif}

La place et le rôle du client dans le collectif doivent être soulignés. II est appelé à changer de position : au-delà de son problème individuel, il découvre l'autre et ses questions, apprend à partager, prend conscience de plusieurs formes possibles de solidarités; il renonce à une relation individuelle avec l'intervenant. II exerce alternativement le rôle d'aidant et celui d'aidé; il offre une possibilité de soutien mutuel sur les plans émotif, informatif, concret et normatif; il découvre la valeur du principe de réciprocité.

\section{Le collectif reste ouvert sur l'extérieur}

En tsg comme en intervention de réseaux, l'attention portée au collectif ne doit pas faire oublier les liens de solidarité avec d'autre groupes ou d'autres réseaux ni les ressources de l'environnement.

\section{Deux visions idéologiques différentes :}

le « dedans » et le « dehors »

Dans le champ du tsg, la finalité du travail est tantôt centrée sur le groupe lui-même, sur le « dedans », tantôt centrée sur une réalisation extérieure, sur le « dehors ».

Dans le premier cas, l'attention est concentrée sur ce qui se passe entre les participants, leurs relations, les sentiments qu'ils 
éprouvent. On se préoccupe du développement de la personne, de la vie intérieure de chacun.

Dans le second, on met l'accent sur la vie sociale du groupe, considéré comme une micro-société; celui-ci parvient parfois à un niveau d'autonomie lui permettant de se mobiliser pour prendre en main une situation collective; ce n'est pas nécessairement l'objectif de départ, c'est un des résultats possibles du travail en commun et des processus qu'il a rendus conscients.

Pour sa part, l'intervention de réseaux est annoncée d'emblée comme un projet d'action sociopolitique. Le réseau (il s'agit ici évidemment du réseau primaire), cellule de base de la société, est appelé à se mobiliser et à se responsabiliser, à s'approprier ses dimensions collectives, à modifier ses rapports avec l'institution. Cette prise en main de la destinée du réseau par lui-même se réalise dans un mouvement constant entre le " dedans " et le " dehors ", entre le clinique et le communautaire, entre le micro- et le macro-social, entre le psychologique et le sociologique. C'est aussi dans ce mouvement que l'individu construit son identité personnelle et sociale, crée les liens avec lui-même et avec la communauté.

Le projet d'action sociopolitique prend une dimension particulière lorsque les membres des réseaux eux-mêmes peuvent reconnaître les retombées des dimensions individuelles des problèmes sur leur collectif et celles des problèmes sociaux à large échelle sur eux-mêmes et sur leur réseau et décider d'une action commune.

\section{Deux atouts qui peuvent devenir écueils}

Entre ces deux pôles du " dedans " et du « dehors ", il convient d'éviter deux excès : d'un côté ce qu'on pourrait appeler la « fascination de la thérapie ", de l'autre celui du " militantisme politique ", ce dernier tout particulièrement dans le domaine de l'intervention de réseaux.

\section{QUELQUES EXEMPLES D'USAGE COMBINÉ DES DEUX MÉTHODES D'INTERVENTION}

Non, dit le petit prince. Je cherche des amis, qu'est-ce que signifie apprivoiser? C'est une chose trop oubliée, dit le renard. Ça signifie créer des liens... Créer des liens? 
Bien sûr, dit le renard. Tu n'es encore pour moi qu'un petit garçon semblable à cent mille petits garçons. Et je n'ai pas besoin de toi. Et tu n'as pas besoin de moi non plus. Je ne suis pour toi qu'un renard semblable à cent mille renards. Mais, si tu m'apprivoises, nous aurons besoin l'un de l'autre. Tu seras pour moi unique au monde. Je serai pour toi unique au monde.

Antoine de Saint-Exupéry

Les exemples qui suivent sont tirés de ma pratique où interagissent plusieurs activités : la coordination d'un service de bénévoles, le partage de la vie quotidienne dans une institution pour personnes déficientes mentales, la formation, la supervision et la pratique en travail social de groupe et en intervention de réseaux.

\section{L'exploration des réseaux personnels au sein d'un groupe}

Un travail en groupe est proposé à quelques femmes étrangères qui vivent une grande solitude et demandent de l'aide à des travailleuses sociales. Le processus s'est déroulé d'une manière tout à fait classique, aussi bien quant au nombre de séances, à leur régularité qu'à la procédure de formation du groupe. En revanche, parmi les moyens utilisés, on a intégré celui qui consiste à dessiner des " cartes de réseaux », support principal permettant de réfléchir et d'exprimer la solitude, dans un premier temps, et de trouver dans un second des pistes de solution.

Les cartes ont permis à chacune des membres une prise de conscience qu'elle était entourée de beaucoup plus de monde qu'elle ne se l'imaginait et que ces ressources n'étaient pas toujours bien utilisées. Chacune a pu repérer aussi les relations qu'elle avait envie d'activer ou de faire cesser.

L'entraide s'est développée au sein du groupe, les réseaux personnels des membres du groupe se sont ouverts les uns aux autres; non seulement virtuellement, mais concrètement; des solidarités de voisinage, quartier par quartier, se sont développées. 


\section{UN EFFET DE LA FORMATION EN GROUPE DES BÉNÉVOLES : OUVERTURE ET MOBILISATION}

Au sein d'un service de solidarité entre femmes, des bénévoles s'engagent à rencontrer régulièrement des femmes en grande difficulté. Elles réalisent chacune un accompagnement amical, le plus souvent de longue durée.

En groupe de formation elles réfléchissent à leur position au sein de leur propre réseau, prennent conscience des personnes qui les entourent, des liens qu'elles établissent, de ceux qu'elles laissent s'étioler, de la fonction de ces liens. Elles saisissent alors l'importance de ce tissu social pour elles en tant que personnes et comme potentiel à partager en tant que bénévoles avec la femme en difficulté, mais aussi avec le service dont elles font partie.

Dans le cadre de l'accompagnement dont elles ont la responsabilité, elles décident de veiller à ne pas isoler encore davantage la personne en difficulté, de rendre possible une réactivation de ses liens, dans la mesure de sa volonté et de ses possibilités.

Elles se mobilisent pour organiser une journée de fête et de formation qu'elles partagent avec les femmes accompagnées et leurs amies.

\section{Une stratégie « réseaux 》 utilisée pour créer un groupe}

Des travailleuses sociales doivent répondre à la demande d'une femme étrangère qui désire apprendre le français. Pour le moment, c'est la seule demande de ce type qu'elles ont reçue.

Elles se demandent dans quelle mesure la solution pourrait être trouvée au sein des réseaux primaires de cette femme. II s'agit d'une personne appartenant à une culture différente où rien ne pourra être entrepris si le mari ne donne pas son accord. Les intervenantes demandent à la jeune femme si, lors de son prochain rendez-vous au service, elle veut bien venir accompagnée de son mari, ce qu'elle accepte. Une question lui est posée au cours de cet entretien: pensez-vous que les épouses de vos amis désireraient également apprendre le français et seriez-vous d'accord de les entretenir de cette idée? Avec son accord, les démarches préalables ayant abouti, un petit groupe est constitué. 
Six jeunes femmes se réunissent régulièrement et apprennent les rudiments du français, d'une façon dynamique, tout en partageant leurs préoccupations et en vivant ensemble une succession d'étapes du groupe.

\section{Un travail avec plusieurs familles : une possibilité de passer de l'intervention de réseaux au tsg}

Une équipe d'éducateurs spécialisés travaillent dans une institution accueillant 180 résidants déficients intellectuels présentant des problèmes divers, dont certains relèvent de la psychiatrie. Dans le pavillon qui les abrite, ils entourent au quotidien un groupe de onze personnes de 19 à 45 ans.

Les relations avec la famille et l'entourage sont qualifiées de "distantes et lacunaires", les occasions d'échanges sont espacées dans le temps, les moyens et les espaces de communication sont restreints, par exemple les parents qui viennent chercher les personnes sont reçus sur le pas de la porte, les communications téléphoniques servent essentiellement à exprimer du mécontentement et à régler des questions problématiques.

Les relations entre les familles et l'équipe éducative se déroulent dans un contexte de réserve réciproque, les informations ont de la peine à circuler, l'équipe éducative connaît peu ou pas l'histoire de chaque famille. Ces dernières ne connaissent rien du groupe, de son histoire ni de son fonctionnement. II y a impossibilité de partager les difficultés de chacun.

Les objectifs des interventions de réseaux ont pris trois directions : maintenir la relation entre les résidants et leur milieu d'origine; améliorer les relations entre les familles et l'entourage des résidants, l'équipe éducative et plus largement les autres services de l'institution; modifier les modes de relation.

Les interventions se sont développées de manières multiples et inattendues, également à l'initiative des résidants et de leur entourage, allant du réseau vers le groupe et du groupe vers le réseau, par exemple :

- les éducateurs se sont rendus dans les réseaux primaires des résidants, sur la proposition de ces derniers et avec les personnes choisies par eux; 
- ils ont invité les membres des réseaux primaires pour des rencontres d'échanges dans leur pavillon;

- certains parents ont constitué un groupe d'entraide à plus long terme et se rencontrent régulièrement dans les locaux de l'institution;

- les résidants ont pris l'initiative d'organiser des fêtes pour réunir les personnes de leur entourage;

- les éducateurs ont pris contact avec des personnes extérieures à l'institution, amis d'atelier, anciens pensionnaires, etc., pour élargir le réseau des personnes handicapées et ils les invitent régulièrement;

- l'équipe éducative pense aujourd'hui à former éventuellement un groupe de bénévoles pour accompagner les personnes handicapées dans la communauté.

\section{EN GUISE DE CONCLUSION : L'“ EFFET RÉSEAU »}

À travers ces exemples on voit la complémentarité et la richesse que suscite l'utilisation combinée du tsg et de l'intervention de réseaux. La force et le dynamisme du collectif renouvellent l'énergie des membres du groupe et du réseau, entraînant également professionnels, familles, entourage et résidants vers des réalisations nécessaires, originales et imprévues.

De nombreuses pistes de réflexions et d'interventions s'ouvrent vers l'avenir.

En terminant, je ne résiste pas au plaisir de citer un très beau passage de Cristina de Robertis qui nous encourage dans la voie d'une toujours meilleure utilisation combinée des deux approches méthodologiques.

L'effet réseau est un phénomène important, en partie verbal et inconscient; qu'accompagne l'ouverture à des interactions de groupe. L'effet réseau se fait sentir dès que les membres ont commencé à réaliser qu'ils font partie d'un groupe humain particulier. Ils ont l'expérience d'un nouveau sentiment de liberté provenant d'une intimité partagée. On peut le comparer à l'espèce d'euphorie, de communion qui se produit lors de cérémonies tribales, de réunions religieuses, de sessions de jazz, etc. 
C'est une expérience excitante qui galvanise le groupe et qui donne assez de confiance aux gens pour qu'ils puissent commencer à s'attaquer à leurs problèmes.

Christiane BESSON

Formatrice

Centre de formation et perfectionnement Améthyste, Lausanne

Chaire de travail social Université de Fribourg

Suisse

\section{Références bibliographiques}

BAHUAUD, A. et collab. (1994), De l'assistance à la solidarisation, un nouveau sens au travail social. Action-recherche collective, Paris, L'Harmattan.

BESSON, Christiane (1994). L'intervention de réseaux, Parcours méthodologique, dans L. Sanicola (dir.), Paris, Bayard Éditions (voir aussi : L. Sanicola [dir.] [1995]. Reti sociali e intervento professionale, Naples, Liguori Editore).

BESSON, Christiane (1983). Une pratique cherche ses racines. Dix ans de travail social de groupe en Suisse romande, Genève, Les Éditions IES, Institut d'Études sociales. (Coll. Champs professionnels, n॰ 7)

BRODEUR, Claude et Richard Rousseau (1984). L'intervention de réseaux, une pratique nouvelle, Montréal, Éditions France-Amérique.

COLLECTIF (1984). Vers des pratiques de réseaux, pour un renouvellement méthodologique en service social, Genève, Les Éditions IES, Institut d'Études sociales. (Coll. Champs professionnels, n 9)

PINCON, Yvonnick (dir.) (1991). L'intervention de réseaux, 20 ans d'expérience, Les Cahiers de l'ARPE n³, Le Bourgneuf-la-Forêt.

DE ROBERTIS, Cristina et Henri PASCAL (1987). L'intervention collective en travail social, l'action auprès des groupes et des communautés, Paris, Le Centurion. (Socioguides)

\section{Revues}

TRANSitions (1989). Pratique de réseau (Les actes du congrès), Val-d'Or, Éditions Meera.

Service social (1990), "Le groupe ici et ailleurs ", vol. 39, no 1, Québec, Université Laval. 